\title{
NeuroAid II (MLC901) and polypharmacy in stroke and the risk of hepatotoxicity: a case report
}

\author{
Anna Misya'il Abdul Rashid ${ }^{1,2^{*}} \oplus$, Mohamad Syafeeq Faeez Md Noh ${ }^{4}$, Abdul Hanif Khan Yusof Khan', \\ Wei Chao Loh', Janudin Baharin ${ }^{1}$, Azliza Ibrahim ${ }^{1}$, Liyana Najwa Inche Mat ${ }^{1}$, Wan Aliaa Wan Sulaiman', \\ Fan Kee Hoo ${ }^{1}$, Fazah Akhtar Hanapiah ${ }^{3}$ and Hamidon Basri ${ }^{1}$
}

\begin{abstract}
Background: NeuroAid II (MLC901) is a promising therapy for stroke patients who present outside of therapeutic window for reperfusion therapy. Studies have proven its efficacy in ischemic stroke; however, data of drug safety and combination with other medications especially anticoagulants are heterogenous. We report a possible case of hepatotoxicity induced by NeuroAid II in combination with anticoagulants.

Case presentation: We report an elderly patient who developed symptoms of cardioembolic stroke presented outside of time window for reperfusion therapy. He was started on a regiment of statins, anticoagulation, beta blockers and NeuroAid II. One month later he presented with deranged liver enzymes. Cessation of NeuroAid II resulted in rapid improvement of transaminitis within days.

Conclusions: We wish to highlight the potential harmful effect of administering NeuroAid II with an anticoagulant and the importance of routine follow-up and blood monitoring in the elderly patients with stroke.
\end{abstract}

Keywords: Stroke, Chinese traditional medication, Hepatotoxicity, NeuroAid II, Elderly

\section{Background}

Neurological disorders cause significant disability and death worldwide, exceeding cardiovascular diseases and malignancies. Among the neurological disorders, stroke contributed the largest proportion of total disabilityadjusted life years (47.3\%) and mortality (67.3\%) [1]. Despite this, therapeutic options are sparse, with thrombolysis limited to a four and a half hours' time window and thrombectomy to the first $24 \mathrm{~h}$ after stroke onset. If a patient presents later, the only modality of treatment is rehabilitation and physical therapy, admission to stroke

\footnotetext{
*Correspondence: annamisyail@yahoo.com

${ }^{1}$ Department of Neurology, Faculty of Medicine and Health Sciences, Universiti Putra Malaysia, 43400 Serdang, Selangor, Malaysia

Full list of author information is available at the end of the article
}

unit as well as decompression craniectomy for malignant middle cerebral artery (MCA) infarcts.

A recent interest has been budding in the field of poststroke neuroplasticity, where a specific gene, namely, the brain derived neurotrophic factor (BDNF), is responsible for neurogenesis, neuronal differentiation and survival in response to cerebral ischemia [2]. A novel drug, MLC 601 (NeuroAiD) and MLC 901 (NeuroAid II), which is a Traditional Chinese Medication (TCM) consisting of nine herbs (Radix astragali, Radix salviae miltiorrhizae, Radix paeoniae rubra, Rhizoma chuanxiong, Radix angelicae sinensis, Carthamus tinctorius, Prunus persica, Radix polygalae and Rhizoma acori tatarinowii) and five animal components (including Hirudo, Eupolyphaga seu steleophaga, Calculus bovisartifactus, Buthus martensii and Cornu saigae tataricae) has demonstrated 
neuroprotective and neuroregenerative effects in brain ischemia $[3,4]$.

Although many efficacy trials relay its safety, reporting very mild side effects, such as dry mouth and gastrointestinal side effects; that resolves after dose reduction [5], data on polypharmacy and combinations with anticoagulants are sparse [6-8]. We would like to share our experience with a case of possible NeuroAid II induced hepatotoxicity when combined with anticoagulants and possible detrimental side effects of polypharmacy.

\section{Case presentation}

A 73-year-old man with underlying dyslipidemia and hypertension was found unconscious at home. $\mathrm{He}$ is a retired engineer, and is living independently on his own. He was not compliant to his antihypertensives and cholesterol medications as he was asymptomatic. He also denied any previous history of liver disease or any drug induced complications prior to this. It was unclear for how long he had been unconscious or unwell as he was not able to recall the chronology of events. Upon assessment, blood pressure was $120 / 80 \mathrm{mmHg}$ with pulse rate of 100 beats per minute. He was noted to have right sided hemiplegia with increased tone and reflexes, dysphagia and dysarthria. His total National Institute of Health Stroke Scale (NIHSS) was ten. Electrocardiogram (ECG) showed atrial fibrillation (AF) and echocardiogram showed good ejection fraction of $76 \%$, moderate left ventricular hypertrophy with normal cardiac chambers and valves. There were multiple grade two and three pressure ulcers on his right temporal region, right anterior chest and right hip, which were not previously present. An urgent magnetic resonance imaging (MRI) of the brain revealed bilateral acute and established pontine infarcts, left more than right with microvascular ischemia in the deep white matter of the brain (Fig. 1). Magnetic resonance angiography (MRA) of the brain showed narrowing of the left posterior cerebral artery, but no evidence of large vessel obstruction (Fig. 2). As he presented late, he was not a candidate for thrombolysis or thrombectomy. Hence, he was admitted for conservative management, rehabilitation and wound care.

Initially he was started on aspirin, which was changed to apixaban five mg twice daily after 14 days of stroke onset. He was also started on atorvastatin $40 \mathrm{mg}$ at night, NeuroAid II two capsules three time daily, beta blockers and proton pump inhibitors. His baseline blood investigations were as follows: hemoglobin $14 \mathrm{~g} / \mathrm{dL}$, platelets $180 \times 10^{9} / \mathrm{L}$, white cell count (WCC) $8.5 \times 10^{9} / \mathrm{L}$, total serum bilirubin (TSB) $10 \mu \mathrm{mol} / \mathrm{L}$, alanine aminotransferase (ALT) $62 \mathrm{IU} / \mathrm{L}$, aspartate transaminase (AST) $75 \mathrm{IU} / \mathrm{L}$, Albumin $27 \mathrm{~g} / \mathrm{L}$. C-reactive protein (CRP) was elevated $134 \mathrm{mg} / \mathrm{L}$. Renal profile showed acute kidney injury (AKI) with urea $10 \mathrm{mmol} / \mathrm{L}$ and creatinine of $123 \mu \mathrm{mol} / \mathrm{L}$.

His pressure ulcers required multiple courses of antibiotics, daily dressings and debridement. It was particularly challenging as it involved a large area and healing was hindered by his thin, aging skin. We postulated that he has been neglected for several days, prior to presentation to cause such an extensive pressure sore. Throughout his stay, he received intravenous cloxacillin and amikacin, piperacillin/tazobactam, cefepime and ampicillin/sulbactam which were appropriate for his tissue cultures. After almost six weeks of tedious care in our ward, his pressure ulcers have improved to grade one, involving a smaller area on his temporals, chest and hip. He was also more alert and was able to tolerate orally. Blood parameters showed resolution of AKI and reduction of CRP to $17 \mathrm{mg} / \mathrm{L}$. He was transferred to a rehabilitation hospital for his stroke recovery with bisoprolol $1.25 \mathrm{mg}$ daily, apixaban five mg twice daily, NeuroAid II two capsules three times daily, atorvastatin $40 \mathrm{mg}$ at night and pantoprazole $40 \mathrm{mg}$ daily.

His baseline blood investigations and liver function tests at the rehabilitation center were normal, ALT was $77 \mathrm{IU} / \mathrm{L}$, ALP $85 \mathrm{IU} / \mathrm{L}$ and TSB $5 \mu \mathrm{mol} / \mathrm{L}$. However, 1 month after the transfer, during a routine wound dressing and follow-up, the liver enzymes were deranged, where his ALT was $146 \mathrm{IU} / \mathrm{L}$, AST $58 \mathrm{IU} / \mathrm{L}$, ALP $77 \mathrm{IU} / \mathrm{L}$ and TSB $8.7 \mu \mathrm{mol} / \mathrm{L}$. He was clinically asymptomatic and was not jaundiced, his blood pressure was $100 / 80 \mathrm{mmHg}$ and pulse rate was 50 beats per minute. Screening for hepatitis $B$ and $C$ were negative. Hence, the statin, beta blocker and pantoprazole were withheld. Low dose digoxin was started as rate-control medication for his AF. An ultrasonography (US) of the hepatobiliary followed by computed tomography (CT) of the abdomen showed a huge simple liver cyst at segments two and three measuring $8.1 \times 6.4 \times 5.6 \mathrm{~cm}$ (Fig. 3). We, therefore, referred him to our surgical colleagues, who offered surgical resection. However, as the family was not keen for surgical intervention, we continued to follow him up to monitor his liver enzymes.

Despite cessation of statin, beta blockers and proton pump inhibitors for two weeks, his liver enzymes continued to increase, ALT was $210 \mathrm{IU} / \mathrm{L}$, AST $85 \mathrm{IU} / \mathrm{L}$. His renal profile was normal, with urea $5.7 \mathrm{mmol} / \mathrm{L}$ and creatinine of $57 \mu \mathrm{mol} / \mathrm{L}$ and CRP was not elevated, at $3.6 \mathrm{mg} / \mathrm{L}$. His pressure sores have very much improved to grade one, blood pressure was $100 / 85 \mathrm{mmHg}$, pulse rate was 55 beats per minute and ECG showed evidence of rate-controlled AF. As the septic parameters were not raised, with no clinical evidence of infection and hemodynamic stability, this precludes our suspicion that the raised transaminitis was due to an infection. Though 


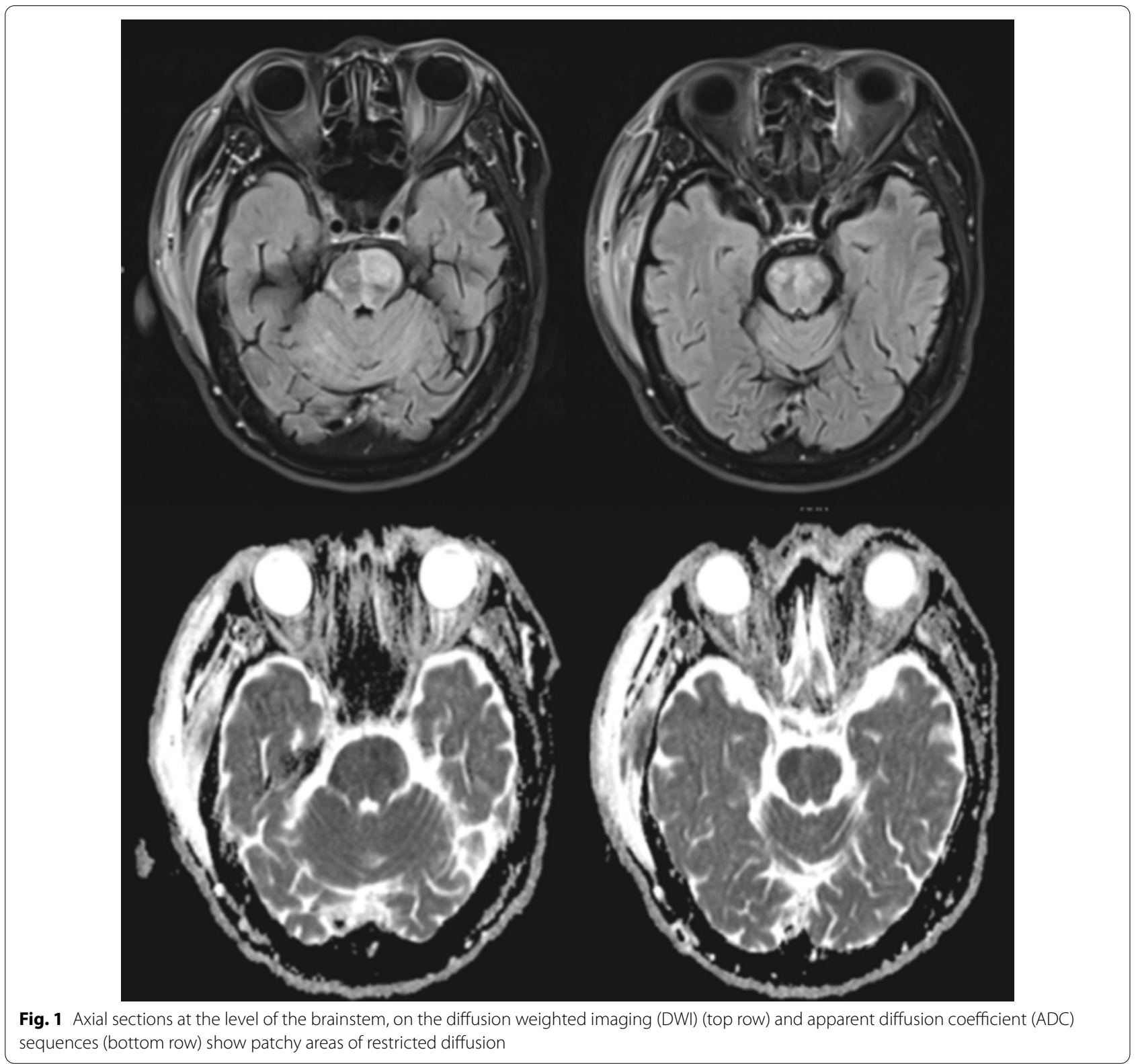

there have been some reports on the derangement of liver enzymes during the acute phase of ischemic stroke; however, they tend to plateau and normalize by day seven, which obviates the transaminitis as an acute phase reactant in our case $[9,10]$. However, due to concerns of worsening liver profiles, we decided a trial of withholding NeuroAid II. Within 5 days, his ALT reduced to $90 \mathrm{IU} / \mathrm{L}$ and AST reduced to $44 \mathrm{IU} / \mathrm{L}$, after 2 weeks of stopping NeuroAid II, his liver enzymes returned to normal. The patient continued to improve and was able to ambulate with a walking frame. As the liver enzymes normalized and patient was asymptomatic, he declined a repeat imaging to reassess the liver cyst.

\section{Discussion}

Acute reperfusion therapy by thrombolysis or thrombectomy is limited to a small subset of patients due to short window of treatment time and several contraindications $[11,12]$. Beyond this time window, treatment for stroke is limited to optimizing medical therapy to prevent future stroke alongside rehabilitation and physiotherapy. However, more recently, in vitro and in vivo studies have shown that NeuroAid II is able to potentially reverse ischemic tissues in the brain and promote neurogenesis [13-15]. Although multiple studies have proved its efficacy and long-term safety profile [16-18], these studies excluded patients 


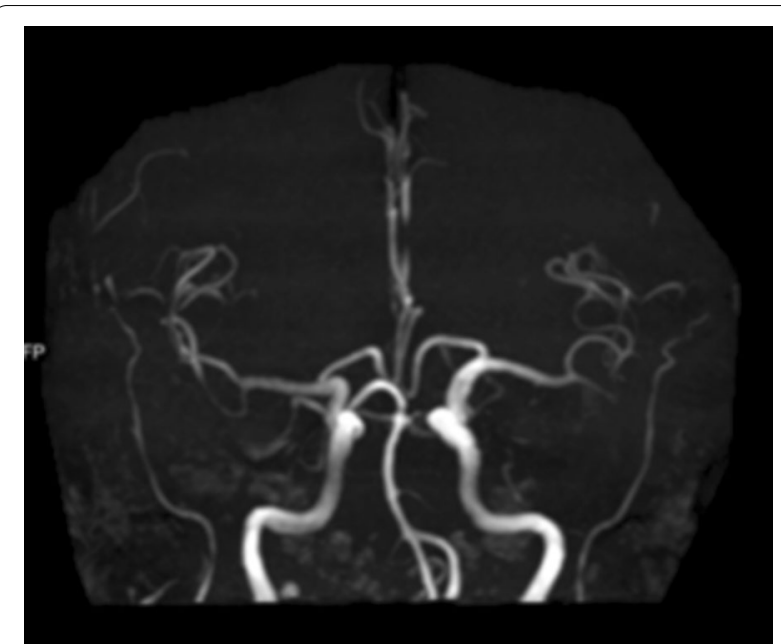

Fig. 2 Magnetic resonance angiography (MRA) shows no evidence of large vessel occlusion

who had concomitant anticoagulation onboard [1921]. As NeuroAid II consist of herbal contents, its use is licensed as Food Supplements as opposed to Drugs in many Asian countries; including Malaysia. Thus, patients' consent is not required for consumption of this drug in our country $[22,23]$.

Despite that, Kong and colleagues showed an alarming trend of hepatotoxicity and jaundice in patients taking NeuroAid II, causing them to withdraw from the study [20]. Other studies demonstrate milder side effects, especially nausea and vomiting [24, 25]. This effect is greatly magnified by several risk factors, such as elderly age group and a more severe stroke [26]. This poses a significant dilemma in treating cardioembolic stroke who present outside of the therapeutic window, as these patients will need indefinite anticoagulation to prevent a recurrence of stroke.

With the emergence of non-vitamin $\mathrm{K}$ antagonist anticoagulant (NOAC) in stroke prevention in AF, warfarin has fallen out of favor [27]. The efficacy and safety of these drugs are well tolerated and they are widely used in clinical practice to date [28]. Major adverse effects with NOACs were bleeding tendencies [29-31]. Although in these trials, there was a small proportion of patients with hepatotoxic effect, it was the same in both NOAC groups as compared to warfarin. There have been several case reports on the hepatotoxicity of NOACs, but they are very rare with a frequency of 0.1$1 \%$. In these reports, hepatotoxicity occurs more often with rivaroxaban as compared to apixaban and dabigatran, although the exact mechanism is still unknown $[32,33]$. Although beta blockers are also associated with deranged liver enzymes [34,35], the fact that we
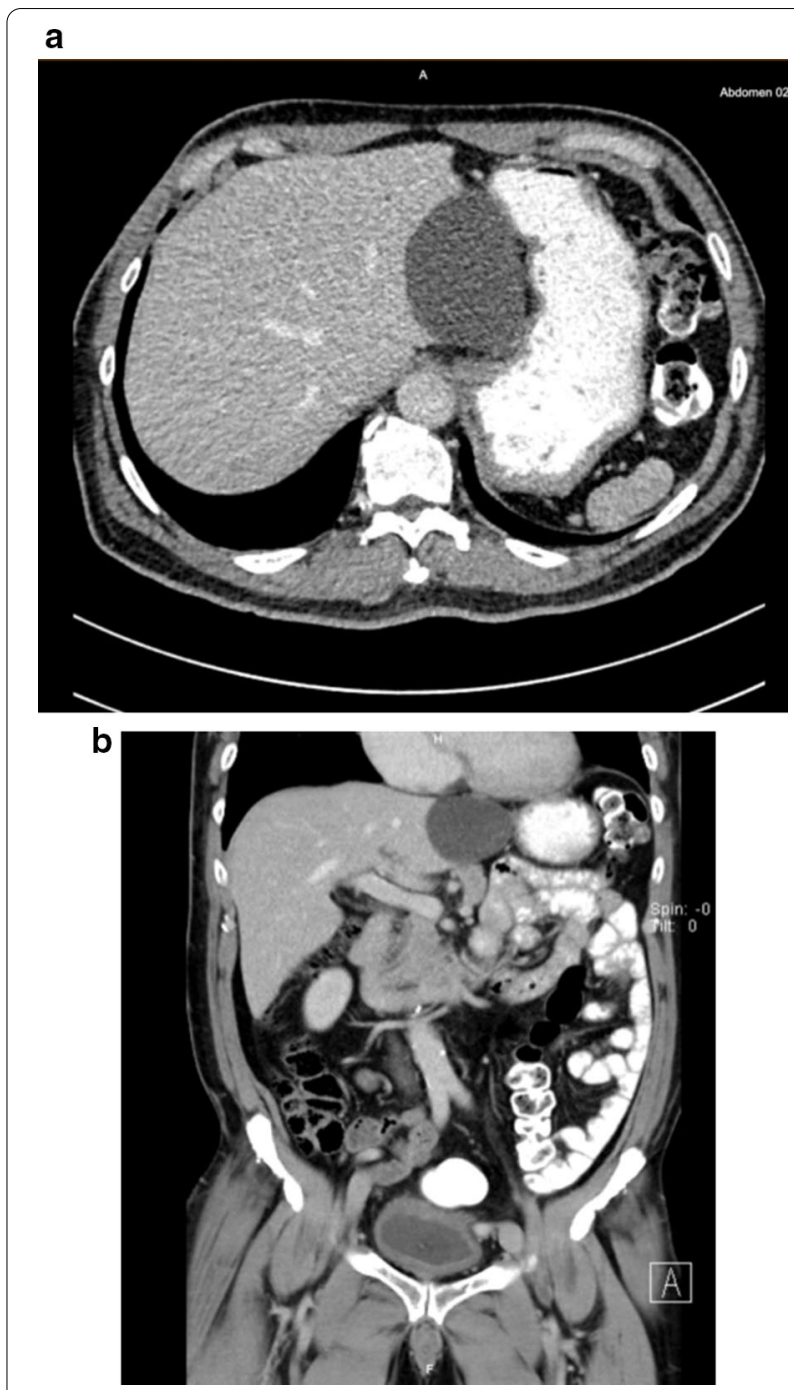

Fig. 3 Axial a and coronal b cut computed tomography (CT) of the abdomen showing a well-defined hypodense mass in segment 2 and 3 (red arrows) of the liver that is suggestive of a simple liver cyst

have stopped the beta blocker with no improvement in liver enzymes ruled the bisoprolol as a causative agent for the transaminitis.

Putting the evidence into perspective, the risk versus benefits in starting medications and co-administration with other drugs needs to be taken into serious consideration. The choice of drug which will provide the best outcome with least side effect should be initiated. In our patient, for instance, the administration of anticoagulant was essential for future stroke prevention due to his underlying AF. As such, we were reluctant to stop his anticoagulation. Furthermore, large trials have proven the safety of NOACs with co administration of multiple drugs [36], which was not fully explored in 
many NeuroAid trials. Therefore, we decided to stop NeuroAid II while maintaining the anticoagulation.

The cessation of NeuroAid II showed a dramatic improvement in the liver function with normalization within 2 weeks of stopping the drug. What's more, the NOAC was successful in preventing the recurrence of stroke and with rehabilitation on board, he was able to ambulate with a walking aid and resume his activity of daily activities with minimal assistance. This was an important outcome for him and his family to achieve, as it improved his quality of life and reduced his dependency. Since the studies on the efficacy of NeuroAid II was not proven to be affective beyond the period of 3 months, it was discontinued indefinitely for our patient.

Most simple liver cysts are benign and present with a picture of cholestasis [37]. Although the size of the liver cyst was big, the CT of the abdomen did not show any evidence of biliary tree obstruction nor did the blood parameters show any evidence of cholestasis. Nonetheless, there is a possibility of the liver cyst causing the transaminitis. In this context, a follow-up imaging of the abdomen was crucial to establish this effect. Unfortunately, we were unable to proceed with a repeat imaging as the patient declined further follow-up as he remained asymptomatic and due to logistics issues. However, due to the temporal relationship on initiating and stopping the Neuroaid II in causing and resolution of transaminitis, we could not confidently rule out the association of NeuroAid II especially in combination with other drugs causing the derangement in liver enzymes.

The hepatotoxicity side effects of TCM has been widely reported, although its causative mechanism and underlying pathophysiology is difficult to determine $[38,39]$. This is due to the fact that these TCMs are manufactured as a combination of several herbal compounds, thus pointing to a particular compound that may cause harmful effects remains a challenge $[40,41]$. Furthermore, these harmful side effects are magnified by patients' factors, such as older age group, more severe stroke and polypharmacy that may worsen the hepatotoxicity.

The strengths of this case report are the new finding of hepatotoxicity of an anticoagulant with NeuroAid II and its strong temporal relation between the resolution of transaminitis with the cessation of the drug. This is important as anticoagulant is the mainstay treatment for cardioembolic stroke. However, the strength of evidence is limited as this is a single case report reporting such association.

\section{Conclusion}

In conclusion, treating stroke in the elderly age group remains a great challenge. Due to higher risk factors and comorbidities, this subgroup is more prone to receive multiple medication for underlying illnesses. The risk of polypharmacy is thus amplified in this population. This case highlights the potential harmful effect of administering NeuroAid with an anticoagulant or polypharmacy and the importance of routine follow-up and blood monitoring in the elderly patients with stroke.

\section{Abbreviations \\ MCA: Middle cerebral artery; BDNF: Brain derived neurotrophic factor; TCM: Traditional Chinese Medication; NIHSS: National Institute of Health Stroke Scale; ECG: Electrocardiogram; AF: Atrial fibrillation; MRI: Magnetic resonance imaging; MRA: Magnetic resonance angiography; WCC: White cell count; TSB: Total serum bilirubin; ALT: Alanine aminotransferase; AST: Aspartate transami- nase; CRP: C-reactive protein; AKI: Acute kidney injury; US: Ultrasonography.}

\section{Acknowledgements}

We would like to thank the Director of Hospital Pengajar Universiti Putra Malaysia for encouraging and allowing the production of this case report.

\section{Authors' contributions}

AHK, WCL and JB was involved in the literature search as well as obtaining consent. AM and FA was involved in the layout and writing of manuscript as well as obtaining clinical information. MSF was involved in the acquisition of the radiographical images. FKH, WA, LA and $\mathrm{HB}$ were involved in critical analysis of the manuscript as well as formatting and editing the manuscript. All authors read and approved the final manuscript.

\section{Funding}

Not applicable.

\section{Availability of data and materials}

The data sets supporting the conclusions of this article are included within the article.

\section{Declarations}

\section{Ethics approval and consent to participate}

The authors' institution does not require ethical approval for publication of a single case report. Written informed consent was obtained from the patient's next of kin (children).

\section{Consent for publication}

Written informed consent was obtained from the patient for publication of this case report and any accompanying images. A copy of the written consent is available for review by the Editor of this journal.

\section{Competing interests}

The authors declare that they have no competing interest.

\section{Author details}

${ }^{1}$ Department of Neurology, Faculty of Medicine and Health Sciences, Universiti Putra Malaysia, 43400 Serdang, Selangor, Malaysia. ${ }^{2}$ Institut Penyelidikan Penuaan Malaysia (MyAgeing ${ }^{\mathrm{TM}}$ ), Universiti Putra Malaysia, 43400 Serdang, Selangor, Malaysia. ${ }^{3}$ Faculty of Medicine, Universiti Teknologi MARA, 47000 Sungai Buloh, Selangor, Malaysia. ${ }^{4}$ Department of Radiology, Faculty of Medicine and Health Sciences, Universiti Putra Malaysia, 43400 Serdang, Selangor, Malaysia.

Received: 26 July 2021 Accepted: 12 October 2021

Published online: 03 November 2021

References

1. Feigin VL, Vos T. Global, regional, and national burden of neurological disorders during 1990-2015: a systematic analysis for the Global Burden of Disease Study 2015. Lancet Neurol. 2017;16(11):877-97. 
2. Coleman ER, Moudgal R, Lang K, Hyacinth HI, Awosika OO, Kissela BM, et al. Early rehabilitation after stroke: a narrative review. Curr Atheroscler Rep. 2017;19(12):59

3. Heurteaux C, Gandin C, Borsotto M, Widmann C, Brau F, Lhuillier M, et al. Neuroprotective and neuroproliferative activities of NeuroAid (MLC601, MLC901), a Chinese medicine, in vitro and in vivo. Neuropharmacology. 2010;58(7):987-1001.

4. Quintard H, Borsotto M, Veyssiere J, Gandin C, Labbal F, Widmann C, et al. MLC901, a traditional Chinese medicine protects the brain against global ischemia. Neuropharmacology. 2011;61(4):622-31.

5. Venketasubramanian N, Kumar R, Soertidewi L, Bakar AA, Laik C, Gan $\mathrm{R}$. The NeuroAiD safe treatment (NeST) registry: a protocol. BMJ Open. 2015:5(11):1-7.

6. Siddiqui FJ, Venketasubramanian N, Chan ESY, Chen C. Efficacy and safety of MLC601 (NeuroAiD ${ }^{\circledR}$ ), a traditional chinese medicine, in poststroke recovery: a systematic review. Cerebrovasc Dis. 2013;35(Suppl 1):8-17.

7. Navarro JC, Molina MC, Baroque AC II, Lokin JK. The use of NeuroAiD (MLC601) in postischemic stroke patients. Rehabil Res Pract. 2012. https:// doi.org/10.1155/2012/506387.

8. Huang S, Lin M, Pan X, Tan Q, Tan K-L. The potential of MLC901 (NeuroAiD $\left.\|^{\mathrm{TM}}\right)$, a traditional Chinese medicine. Neurosci Res Notes. 2019;2(2):18-24.

9. Muscari A, Collini A, Fabbri E, Giovagnoli M, Napoli C, Rossi V, et al. Changes of liver enzymes and bilirubin during ischemic stroke: mechanisms and possible significance. BMC Neurol. 2014;14(1):1-8.

10. Ruban A, Daya N, Schneider ALC, Gottesman R, Selvin E, Coresh J, et al. Liver enzymes and risk of stroke: the atherosclerosis risk in communities (aric) study. J Stroke. 2020;22(3):357-68.

11. Nogueira RG, Jadhav AP, Haussen DC, Bonafe A, Budzik RF, Bhuva P, et al. Thrombectomy 6 to 24 hours after stroke with a mismatch between deficit and infarct. N Engl J Med. 2018;378(1):11-21.

12. Albers GW, Marks MP, Kemp S, Christensen S, Tsai JP, Ortega-Gutierrez S, et al. Thrombectomy for stroke at 6 to 16 hours with selection by perfusion imaging. N Engl J Med. 2018;378(8):708-18.

13. Widmann C, Gandin C, Petit-Paitel A, Lazdunski M, Heurteaux C. The Traditional Chinese Medicine MLC901 inhibits inflammation processes after focal cerebral ischemia. Sci Rep. 2018;8(1):1-15.

14. Heurteaux C, Widmann C, Moha ou Maati H, Quintard H, Gandin C, Borsotto M, et al. NeuroAiD: properties for neuroprotection and neurorepair. Cerebrovasc Dis. 2013;35:1-7.

15. Chan HYA, Stanton LW. A pharmacogenomic profile of human neural progenitors undergoing differentiation in the presence of the traditional Chinese medicine NeuroAiD. Pharmacogenomics J. 2016:16(5):461-71.

16. Navarro JC, Gan HH, Lao AY, Baroque AC, Hiyadan JH, Chua CL, CHIMES Study Investigators, et al. Baseline characteristics and treatment response of patients from the Philippines in the CHIMES study. Int J Stroke. 2014:9:102-5.

17. Harandi AA, Abolfazli R, Hatemian A, Ghragozlee K, Ghaffar-Pour M, Karimi M, et al. Safety and efficacy of MLC601 in Iranian patients after stroke: a double-blind placebo-controlled clinical trial. Stroke Res Treat. 2011;2011:721613.

18. Young $\mathrm{SH}$, Zhao Y, Koh A, Singh R, Chan BP, Chang HM, et al. Safety profile of MLC601 (Neuroaid) in acute ischemic stroke patients: A Singaporean substudy of the Chinese medicine neuroaid efficacy on stroke recovery study. Cerebrovasc Dis. 2010;30(1):1-6.

19. Chen $\mathrm{CL}$, Venketasubramanian $\mathrm{N}$, Lee CF, Wong KS, Bousser MG. Effects of MLC601 on early vascular events in patients after stroke: the CHIMES study. Stroke. 2013;44(12):3580-3.

20. Kong KH, Wee SK, Ng CY, Chua K, Chan KF, Venketasubramanian N, et al. A double-blind, placebo-controlled, randomized phase II pilot study to investigate the potential efficacy of the traditional chinese medicine Neuroaid (MLC 601) in enhancing recovery after stroke (TIERS). Cerebrovasc Dis. 2009;28(5):514-21.

21. Shahripour BR, Shamsaei G, Pakdaman H, Mojdinasab N, Nejad EM, Sajedi $\mathrm{SA}$, et al. The effect of NeuroAiD ${ }^{\mathrm{TM}}$ (MLC601) on cerebral blood flow velocity in subjects' post brain infarct in the middle cerebral artery territory. Eur J Intern Med. 2011;22(5):509-13.

22. Monthly Index of Medical Specialities. Malaysia. 2021. https://www.mims. com/malaysia/drug/info/neuroaid. Accessed 7 Sep 2021.
23. National Pharmaceutical Control Bureau, Ministry of Health Malaysia. Malaysian Variation Guideline for Natural (Traditional Medication and Homeopathy) and Health Care Supplement Products. 2016. https://www. npra.gov.my/index.php/en/guidelines-for-natural-products/1530-malay sian-variationguideline-for-natural-traditional-medicine-homeopathyand-health-supplement-product-2016.html. Accessed 12 Oct 2016.

24. Chen C, Venketasubramanian N, Gan RN, Lambert C, Picard D, Chan BPL, et al. Danqi Piantang Jiaonang (DJ), a traditional Chinese medicine, in poststroke recovery. Stroke. 2009;40(3):859-63.

25. Ghandehari K, Mood ZI, Ebrahimzadeh S, Picard D, Zhang Y. NeuroAid (MLC601) versus piracetam in the recovery of post-infarct homonymous hemianopsia. Neural Regen Res. 2011;6(6):418-22.

26. Venketasubramanian N, Moorakonda RB, Lu Q, Chen CLH. Frequency and clinical impact of serious adverse events on post-stroke recovery with NeuroAiD (MLC601) versus Placebo: the CHInese medicine neuroaid efficacy on stroke recovery study. Cerebrovasc Dis. 2020;49(2):192-9.

27. Schwill S, Krug K, Peters-Klimm F, van Lieshout J, Laux G, Szecsenyi J, et al. Novel oral anticoagulants in primary care in patients with atrial fibrillation: a cross-sectional comparison before and after their introduction. BMC Fam Pract. 2018. https://doi.org/10.1186/s12875-018-0796-4.

28. Kirchhof P, Benussi S, Kotecha D, Ahlsson A, Atar D, Casadei B, et al. 2016 ESC Guidelines for the management of atrial fibrillation developed in collaboration with EACTS. Eur Heart J. 2016;37(38):2893-962.

29. Granger CB, Alexander JH, MCMurray JJV, Lopes RD, Hylek EM, Hanna M, et al. Apixaban versus Warfarin in patients with atrial fibrillation. N Engl J Med. 2011;365(11):981-92.

30. Connolly SJ, Ezekowitz MD, Yusuf S, Eikelboom J, Oldgren J, Parekh A, et al. Dabigatran versus Warfarin in patients with atrial fibrillation. N Engl J Med. 2009;361:1139-51.

31. Patel MR, Mahaffey KW, Garg J, Pan G, Singer DE, Hacke W, et al. Rivaroxaban versus warfarin in nonvalvular atrial fibrillation. N Engl J Med. 2011;365(10):883-91.

32. Liakoni E, Rätz Bravo AE, Krähenbühl S. Hepatotoxicity of new oral anticoagulants (NOACs). Drug Saf. 2015;38(8):711-20.

33. Maura G, Bardou M, Billionnet C, Weill A, Drouin J, Neumann A. Oral anticoagulants and risk of acute liver injury in patients with nonvalvular atrial fibrillation: a propensity-weighted nationwide cohort study. Sci Rep. 2020;10(1):1-11.

34. Rua J, Prata AR, Marques R, Silva R, Gomes B, Fraga J, et al. Carvedilolinduced liver injury, a rare cause of mixed hepatitis: a clinical case. GE Port J Gastroenterol. 2019;26(3):196-201.

35. Philips C, Paramaguru R, Mahadevan P, Ravindranath J, Augustine P. Metoprolol-induced severe liver injury and successful management with therapeutic plasma exchange. Cureus. 2017. https://doi.org/10.7759/ cureus.1209.

36. Alings M. Individualising anticoagulant therapy in atrial fibrillation patients. Arrhythm Electrophysiol Rev. 2016;5(2):102-9.

37. Mavilia MG, Pakala T, Molina M, Wu GY. Differentiating cystic liver lesions: a review of imaging modalities, diagnosis and management. J Clin Transl Hepatol. 2018;6(2):208-16.

38. Jing J, Teschke R. Traditional Chinese medicine and herb-induced liver injury: comparison with drug-induced liver injury. J Clin Transl Hepatol. 2018;6(1):57-68.

39. Amadi CN, Orisakwe OE. Herb-induced liver injuries in developing nations: an update. Toxics. 2018;6(2):28.

40. Wu Q, Cai C, Guo P, Chen M, Wu X, Zhou J, et al. In silico Identification and mechanism exploration of hepatotoxic ingredients in traditional Chinese medicine. Front Pharmacol. 2019:10:1-15.

41. Teschke R, Eickhoff A. Herbal hepatotoxicity in traditional and modern medicine: Actual key issues and new encouraging steps. Front Pharmacol. 2015;6:1-40.

\section{Publisher's Note}

Springer Nature remains neutral with regard to jurisdictional claims in published maps and institutional affiliations. 